\title{
BMJ Global Health Physicochemical equivalence of generic antihypertensive medicines (EQUIMEDS): protocol for a quality of medicines assessment
}

\author{
Julie Redfern, ${ }^{1}$ Rufus Adesoji Adedoyin, ${ }^{2}$ Sandra Ofori, ${ }^{3}$ Raghupathy Anchala,,${ }^{4,5}$ \\ Vamadevan S Ajay, ${ }^{5}$ Luciano De Andrade, ${ }^{6}$ Jose Zelaya, ${ }^{7}$ Harparkash Kaur, ${ }^{8}$ \\ Dina Balabanova, ${ }^{9}$ Mahmoud U Sani ${ }^{10}$
}

To cite: Redfern J,

Adedoyin RA, Ofori $\mathrm{S}$, et al. Physicochemical equivalence of generic antihypertensive medicines (EQUIMEDS): protocol for a quality of medicines assessment. BMJ Global Health 2016;1: e000086. doi:10.1136/ bmjgh-2016-000086

Received 9 May 2016 Revised 2 August 2016 Accepted 27 August 2016

CrossMark

For numbered affiliations see end of article.

\section{Correspondence to}

Professor Julie Redfern; jredfern@georgeinstitute. org.au

\section{ABSTRACT}

Background: Prevention and optimal management of hypertension in the general population is paramount to the achievement of the World Heart Federation (WHF) goal of reducing premature cardiovascular disease (CVD) mortality by $25 \%$ by the year 2025 and widespread access to good quality antihypertensive medicines is a critical component for achieving the goal. Despite research and evidence relating to other medicines such as antimalarials and antibiotics, there is very little known about the quality of generic antihypertensive medicines in low-income and middleincome countries. The aim of this study was to determine the physicochemical equivalence (percentage of active pharmaceutical ingredient, API) of generic antihypertensive medicines available in the retail market of a developing country.

Methods: An observational design will be adopted, which includes literature search, landscape assessment, collection and analysis of medicine samples. To determine physicochemical equivalence, a multistage sampling process will be used, including (1) identification of the 2 most commonly prescribed classes of antihypertensive medicines prescribed in Nigeria; (2) identification of a random sample of 10 generics from within each of the 2 most commonly prescribed classes; (3) a geographical representative sampling process to identify a random sample of 24 retail outlets in Nigeria; (4) representative sample purchasing, processing to assess the quality of medicines, storage and transport; and (5) assessment of the physical and chemical equivalence of the collected samples compared to the API in the relevant class. In total, 20 samples from each of 24 pharmacies will be tested (total of 480 samples).

Discussion: Availability of and access to quality antihypertensive medicines globally is therefore a vital strategy needed to achieve the WHF $25 \times 25$ targets. However, there is currently a scarcity of knowledge about the quality of antihypertensive medicines available in developing countries. Such information is important for enforcing and for ensuring the quality of antihypertensive medicines.
Summary Box

- This protocol outlines an observational study that includes identification, assessment, collection and analysis of medicine samples.

- Availability of and access to quality antihypertensive medicines globally is a vital strategy needed to achieve the World Heart Federation $25 \times 25$ target.

- This research will inform evidence about the quality of antihypertensive medicines available in a developing country.

- This evidence will directly inform decisionmakers about the potential need for implementation of mechanisms to ensure availability of quality medicines to those who need it.

\section{BACKGROUND}

Elevated blood pressure (BP), often termed hypertension, is one of the most important public health problems worldwide affecting 1 billion people, three-quarters of which live in the low-income and middle-income countries. ${ }^{1}$ High BP is also the leading cause of cardiovascular disease (CVD) and is responsible for $13 \%$ of deaths globally. ${ }^{2}$ Indeed, high BP is second, after smoking, as a contributor to the global burden of disease in the most recent analysis. ${ }^{3}$ In most individuals, adequate management and control of BP is associated with a reduction in deaths and disability from a number of conditions, including cerebrovascular, cardiovascular and renal disease. ${ }^{4}$ Therefore, prevention and optimal management of high BP in the general population is paramount to the achievement of the World Heart Federation (WHF) goal of reducing premature CVD mortality by $25 \%$ by the year 2025 and widespread access to good quality antihypertensive medicines is a critical component for achieving the goal. ${ }^{5}$ 
In recent years, it has emerged that the availability of substandard medicines is widespread and is a crucial public health problem in developing countries. In these countries, half of the medicines for major diseases such as malaria have been found to be substandard in quality and often have little or no active ingredient. ${ }^{6}$ Substandard medicines are defined as medicines that are produced with inadequate attention to good manufacturing practices and may have contents or dissolution times that are outside the pharmacopoeia accepted limits. ${ }^{6-10}$ The problem of modern-day substandard medicines was first highlighted in 1985, and according to $\mathrm{WHO}, 25 \%$ of all medicines available in developing countries are substandard. ${ }^{11}$ Substandard generic medicines are a major contributor and have been shown to constitute 40-50\% of all medicine supplies in Nigeria, ${ }^{12}$ one-third of all antibiotics and antimalarial medicines in Nigeria and Thailand, and 9\% of medicines in India. ${ }^{13}$ Substandard generic medicines share a huge market as estimated by WHO at more than US\$35 billion which represents $>15 \%$ of the pharmaceutical market worldwide. ${ }^{14}$

Almost all kinds of medicines can be substandard; however, most existing studies have investigated antimalarial medicines and antibiotics. The US Pharmacopoeia (USP) drug quality and information programme listed antihypertensive medicines among a class of possible substandard medicines in the African region. ${ }^{14}$ A study carried out in Philippines found that the antihypertensive medicine Adalat (Nifedipine) was one of the top five substandard medications. ${ }^{15}$ However, there is currently very little research being performed to assess the quality of generic antihypertensive medicines. Importantly, given that people with high BP often require lifelong adherence to indicated medicines, the problem of substandard antihypertensive medicines is potentially a serious public health issue. Therefore, the aim of this pilot study was to determine the physicochemical equivalence (percentage of active pharmaceutical ingredient (API)) of generic antihypertensive medicines available in the retail market of Nigeria.

\section{METHODS/DESIGN}

An observational study design will be adopted, which includes searching publically available data relating to antihypertensive medicines in Nigeria as well as collection and laboratory analysis of medicine samples. Technical aspects of the methodology are based on integrated recommendations from published guidelines and recommendations. ${ }^{16} 17$ To determine physicochemical equivalence, a multistage sampling process will be used, including (1) identification of the two most commonly prescribed classes of antihypertensive medicines prescribed in Nigeria; (2) identification of a random sample of 10 generic medicines from within each of the two most commonly prescribed classes; (3) a geographical sampling process to identify a random sample of 24 retail outlets in Nigeria; (4) sample purchasing, processing, storage and transport; and (5) assessment of the physical and chemical equivalence of the collected samples compared to the active drug in the relevant class.

\section{Context}

Nigeria was chosen as the country in which medicines will be collected for this study. Nigeria is a developing country with a large population with previously demonstrated availability of substandard antimalarial medicines. ${ }^{18}$ The prevalence of hypertension in Nigeria is currently estimated to be $28.9 \%$ and this contributes a substantial proportion of the total burden in Africa due to the large population of the country which is currently estimated to be over 170 million. ${ }^{19}$ This increasing burden is increasing the need for government and other health policy planners to ensure the availability of highquality evidence-based medicines for hypertension. ${ }^{20}$ Nigeria has an area of $923768 \mathrm{~km}^{2}$ (about 574000 square miles).$^{21}$ It is the most densely populated country in Africa and represents about $20 \%$ of the total population of sub-Saharan Africa. ${ }^{21}$ Although Nigeria has a large number of ethnic groups $(\sim 250)$, the three most populous are the Hausas in the North, Yorubas in the West and Ibos in the Eastern part of Nigeria. Nigeria has a total of 36 states and 774 local government areas (LGAs) that are subordinate to the relevant states and the Federal Capital Territory. According to the WHO, over half a million Nigerians died from noncommunicable diseases, including CVDs, in 2012 and that $35 \%$ of Nigerian adults had elevated BP in $2008 .{ }^{22}$ Other population-based research reports that the prevalence of hypertension in Nigeria is around 23\% of the adult population with rates being higher in urban than in rural dwellers $(32.7 \%$ and $12.9 \%) \cdot{ }^{23}$ For this study, we have purposively selected a state from three geopolitical zones.

\section{Identification of antihypertensive classes for collection}

To identify which generic brands to include for sampling, we initially identified the most commonly prescribed brand and antihypertensive for each of the five primary classes of antihypertensive medicines available in Nigeria (table 1). The major classes identified were (calcium channel blockers, $\beta$ blockers, ACE inhibitors, angiotensin receptor blockers and centrally acting drugs) based on information obtained from local opinion leaders and from publically available information via the National Agency Food and Drug Administration and Control (NAFDAC) agency. ${ }^{24}$ Based on the classes and brands identified (listed in table 1), a detailed and comprehensive list of all the available generic brands was prepared based on data publically available through NAFDAC. ${ }^{24}$ It was decided that the project would focus on the two most commonly prescribed antihypertensive classes and these were amlodipine and lisinopril. A computerised random sequence was then used to randomly select 10 generic brands of amlodipine and lisinopril. These 20 generic medicines 
Table 1 Most commonly prescribed antihypertensive medicines available in Nigeria*

\begin{tabular}{lll}
\hline $\begin{array}{l}\text { Medication } \\
\text { class }\end{array}$ & $\begin{array}{l}\text { Most commonly } \\
\text { prescribed brand }\end{array}$ & $\begin{array}{l}\text { Number of } \\
\text { generics available } \\
\text { in Nigeria }\end{array}$ \\
\hline $\begin{array}{l}\text { Calcium } \\
\text { channel blocker }\end{array}$ & Amlodipine & 187 \\
$\beta$ blockers & Atenolol & 52 \\
ACE inhibitors & Lisinopril & 92 \\
\hline ARBs & Losartan & 29 \\
\hline $\begin{array}{l}\text { Centrally acting } \\
\text { drugs }\end{array}$ & Methyldopa & 75 \\
\hline
\end{tabular}

*Based on data available on the NAFDAC website http://www. nafdac.gov.ng and information obtained from local experts. ARB, angiotensin receptor blocker; NAFDAC, National Agency Food and Drug Administration and Control.

are available in Nigeria and will form the basis of the samples for collection.

\section{Geographical sampling framework}

A stepwise and semi-random sampling framework was developed to determine which retail outlets would eventually be included in the study. The purpose and concept behind the sampling framework is to identify a combination of urban and rural locations in northern and southern states of Nigeria via a stratified yet random and systematic approach. The steps involved in the sampling framework are as follows:

1. Identification of three states representing varied geopolitical areas through consultation with local experts (purposive sample).

2. For each of the three states, we will then obtain a list of LGAs from which we will randomly select one rural and one urban LGA. Therefore, across the three states, a total of six LGA will be included for sampling (three rural and three urban).

3. It was not feasible to source a list of all pharmacies in each of the LGA, so we will use geographical mapping software (QGIS Geographic Information System, V.2.8.3-Wien, 2015) to randomly select four geographical coordinates in each of the selected LGAs.

4. The nearest available pharmacy outlet to each of the geographical coordinates will then be identified by the research assistant who will act as a mystery shopper and purchase the predetermined generic medicines from each shop. The total sample will therefore include 24 retail outlets (pharmacy shops) from six LGAs across three states in different geopolitical zones of Nigeria.

\section{Medicine purchasing, processing storage and transport}

Once the final list of 24 retail outlets is confirmed, a research assistant will act as a mystery shopper (a hired research assistant who speaks the local language) who will visit the identified outlet and systematically purchased all the available and preselected generic brands for each of the chosen antihypertensive medicines. The covert approach is to minimise the risk that a seller might realise they are to be a part of a research project and therefore raise suspicion and minimise the risk that the seller declines to sell the required samples. Trained research assistants within our existing networks will collect the medicine samples from the pharmacies and will also record the exact geographical coordinates of the pharmacy. Researchers will pose as clients to buy the 20 samples from each of the 24 pharmacy shops (total of 480 samples). A full box (usually 28 or 30 tablets) for each generic medicine will be purchased so the number of dosage units in a box will be the number purchased.

Once purchased, each sample will be retained in their point-of-purchase packaging and placed in an individual zip-lock bag together with a completed standardised data collection form. The data collection form for each sample will include recording of the outlet type, date of purchase, price paid, brand name, formulation, batch number, manufacturer, number of tablets per sample and expiry date. Samples will then be safely and securely stored in an air-conditioned room $\left(\sim 20^{\circ} \mathrm{C}\right)$ in a central location at one of our researcher's sites, pending dispatch. As such, efforts will be made to ensure that the medicines are not degraded by inadequate storage conditions, which could confound the findings. As soon as all samples have been collected, they will be dispatched, via international courier (eg, DHL Express), to the London School of Hygiene and Tropical Medicine (LSHTM) in London, UK for further processing and chemical content analysis.

\section{Sample processing and laboratory analyses}

For every sample under investigation, a digital photograph of the packaging and contents of each sample will be taken. Sample information (including name, stated active ingredients, dose form, outlet, district, date of purchase, manufacturer, NAFDAC registration number, batch number, expiry date, number of tablets per packet and price paid) will be compared to the data collected for each sample during the fieldwork collection and logged on an excel sheet. The integrity of the packaging, the features of the blister pack with indented or printed batch number, and dates of manufacture and expiry, as well as the appearance of tablets, form the first steps in determining the quality of the product. Included on the package should be the list of active ingredients, name and address of the manufacturer, storage conditions, batch or lot number, date of manufacture and directions for use. Enclosed with the tablets should be an instruction leaflet in the appropriate language without any spelling errors. All tablets will then be weighed, and their dimensions, colour and physical appearance characteristics (such as any cracks, logos and chips) recorded prior to laboratory analysis. 


\section{Chemical content analysis}

Quantitative analysis will be conducted at the LSHTM to measure the amount of stated API in the tablets using high-performance liquid chromatography (HPLC). We will use the published methods in the USP monographs for the analysis of tablets of amlodipine besylate and those of lisinopril. ${ }^{25}{ }^{26}$ Tablets will be dissolved in a solvent, sonicated and centrifuged. The supernatant will then be injected into the HPLC column and the amount of API present in each tablet will be determined and expressed as percentage found. We will also consult USP monographs for the two antihypertensive medicines to see what the tolerance rules stipulate which is generally considered acceptable quality if the API measured is $90-110 \%$ for amlodipine besylate tablets and $98-102 \%$ for lisinopril tablets.

\section{Sample size and analysis}

Laboratory data will be combined with sample data and analysed using STATA (Stata/IC). Descriptive statistics will be used to compare the generic medicines on their physical and chemical characteristics with the reference product (medicine). In addition, we will compare equivalence in rural versus urban areas. Proportions will be evaluated using $\chi^{2}$ tests and associations between poor quality and high-quality antihypertensive medicines will be explored. The USP recommends 30 dosage units for a single tablet/capsule medicine of the same lot number from each location. Such a sample size gives enough dosage units to determine identity and accurate estimates of content of active ingredients.

\section{Ethics approvals}

The study will seek ethical approval from the LSHTM Ethics Committee and the relevant Nigerian state-based ethics committees. This study does not involve patients and hence written informed consent will not be required.

\section{DISCUSSION}

BP lowering is one of the priority areas identified for action by the WHF if the target of a $25 \%$ reduction in premature CVD mortality by 2025 is to be achieved. This research will allow the generation of evidence on the presence of substandard antihypertensive medicines among generic antihypertensive medicines in a developing country. This is anticipated to sensitise policymakers, national drug regulatory authorities and governments to the issue of substandard medicines and facilitate debate among health providers, national cardiology societies/ associations and civil society representatives. Ultimately, optimising antihypertensive medicine quality is likely to reduce the overall cardiovascular burden in Nigeria.

This proposal ultimately aims to improve access to good quality antihypertensive medicines in low-income and middle-income countries and is therefore directly linked to health service requirements needed to achieve successful implementation of effective BP control. To the best of our knowledge, no studies have been performed in Nigeria (and other low-income and middle-income countries) regarding the quality of the available generic antihypertensive medicines. This pilot study will (1) allow us to generate evidence on the presence of substandard antihypertensive medicines (based on the physicochemical equivalence) among generic antihypertensive medicines in a low-income and middle-income country; (2) help to sensitise the policymakers, national drug regulatory authorities and key regional and national governments; and finally (3) allow a debate among health providers, national cardiology societies/associations and civil society representatives. Future research will be needed to expand the sampling framework, including branded medicines, more samples and more locations in more countries.

For this research, we have limited the chosen areas of Nigeria due to previous research related to substandard antimalarial medicines and also for practical reasons. However, it is important to acknowledge that this is a small step aimed at catalysing debate related to the quality of antihypertensive medicines available in developing countries. This pilot study is also limited by the nature of the sampling framework (although randomised); it will, however, inform future research and optimal sampling as well as practical barriers and enablers to this type of research. In addition, this research is limited to generic medicines only and future research should also include branded medicines. In this initial research, we will not involve the national regulator given their role and impact remains unclear. However, the research team will liaise with and gain support of local health officials to ensure governance during sample collection.

\section{Author affiliations}

${ }^{1}$ The George Institute for Global Health, Sydney Medical School, University of Sydney, Camperdown, New South Wales, Australia

${ }^{2}$ Department of Medical Rehabilitation, College of Health Sciences, Obafemi Awolowo University, lle-lfe, Nigeria

${ }^{3}$ Department of Internal Medicine, University of Port Harcourt and University of Port Harcourt Teaching Hospital, Choba, Rivers State, Nigeria

${ }^{4}$ Department of Epidemiology, Indian Institute of Public Health, Hyderabad, India

${ }^{5}$ Public Health Foundation of India (PHFI), Gurgaon, Haryana, India

${ }^{6}$ Department of Medicine, State University of Maringa, University Hospital,

Maringá, Brazil

${ }^{7}$ Peruvian Society of Hypertension, Lima, Peru

${ }^{8}$ London School of Hygiene \& Tropical Medicine, London, UK

${ }^{9}$ Department of Global Health and Development, London School of Hygiene \& Tropical Medicine, London, UK

${ }^{10}$ Department of Medicine, Bayero University Kano \& Aminu Kano Teaching Hospital, Kano, Kano State, Nigeria

Handling editor Seye Abimbola

Twitter Follow Julie Redfern at @JRedHeart

Acknowledgements The authors thank the World Heart Federation Emerging Leader Program and Committee who supported and peer reviewed the 
content of this research. In particular, the authors thank Mark Huffman and Pablo Perel for their support and enthusiasm.

Contributors All authors conceived and developed this research as a collaborative group during international workshop and subsequent meetings through the World Heart Federation Emerging Leader Program. RAA, SO and MS are leading the Nigerian component and HK led the development of the sample analysis process. JR, RAA, ASV, LDA, JZ and DB provided leadership regarding sampling, data collection, sample size and geographical mapping. All authors have reviewed and approved the final manuscript.

Funding This research is funded by a World Heart Federation Emerging Leader Program pilot funding grant.

Competing interests None declared.

Provenance and peer review Not commissioned; externally peer reviewed.

Data sharing statement No additional data are available.

Open Access This is an Open Access article distributed in accordance with the Creative Commons Attribution Non Commercial (CC BY-NC 4.0) license, which permits others to distribute, remix, adapt, build upon this work noncommercially, and license their derivative works on different terms, provided the original work is properly cited and the use is non-commercial. See: http:// creativecommons.org/licenses/by-nc/4.0/

\section{REFERENCES}

1. Ibrahim MM, Damasceno A. Hypertension in developing countries. Lancet 2012;380:611-9.

2. World Health Organization. Global health risks: mortality and burden of disease attributable to selected major risks. Geneva: World Health Organization, 2009.

3. Lim SS, Vos T, Flaxman AD, et al. A comparative risk assessment of burden of disease and injury attributable to 67 risk factors and risk factor clusters in 21 regions, 1990-2010: a systematic analysis for the Global Burden of Disease Study 2010. Lancet 2012;380:2224-60.

4. Lewington S, Clarke R, Qizilbash N, et al. Age-specific relevance of usual blood pressure to vascular mortality: a meta-analysis of individual data for one million adults in 61 prospective studies. Lancet 2002;360:1903-13.

5. Beran D, Perrin C, Billo N, et al. Improving global access to medicines for non-communicable diseases. Lancet Glob Health 2014;2:e561-2.

6. Attaran A, Barry D, Basheer S, et al. How to achieve international action on falsified and substandard medicines. BMJ 2012;345: e7381.

7. Cockburn R, Newton PN, Agyarko EK, et al. The global threat of counterfeit drugs: why industry and governments must communicate the dangers. PLoS Med 2005;2:e100.
8. Newton PN, McGready R, Fernandez F, et al. Manslaughter by fake artesunate in Asia-will Africa be next? PLoS Med 2006;3:e197.

9. Newton PN, Green MD, Fernández FM, et al. Counterfeit anti-infective drugs. Lancet Infect Dis 2006;6:602-13.

10. Keoluangkhot V, Green MD, Nyadong L, et al. Impaired clinical response in a patient with uncomplicated falciparum malaria who received poor-quality and underdosed intramuscular artemether. $A m$ J Trop Med Hyg 2008;78:552-5.

11. World Health Organization. Media centre, 2015. http://www.who.int/ mediacentre/factsheets/fs275/en/ (accessed 26 Mar 2015).

12. Almuzaini T, Choonara I, Sammons H. Substandard and counterfeit medicines: a systematic review of the literature. BMJ Open 2013;3: e002923.

13. Banerjee $Y$. Mission. Indradhanush and the counterfeit drug trade in India. Lancet Infect Dis 2015;15:1379-80.

14. Morris J, Stevens P. Counterfeit medicines in less developed countries: problem and solutions. International Policy Network, 2006

15. World Health Professions Alliance. Background document on counterfeit medicines in Asia, 2011. http://www.whpa.org/ Background_document_Counterfeit_Medicines_in_Asia.pdf (accessed 2 Aug 2016).

16. World Health Organisation. Guidelines on the conduct of surveys of the quality of medicines, June 2015. http://www.who.int/medicines/areas/ quality_safety/quality_assurance/Guidelines-on-medicines-qualitysurveys-QAS15-630_30062015.pdf (accessed 29 Jun 2016).

17. Newton PN, Lee SJ, Goodman C, et al. Guidelines for field surveys of the quality of medicines: a proposal. PLoS Med 2009;6:e52.

18. Kaur H, Allan EL, Mamadu I, et al. Quality of artemisinin-based combination formulations for malaria treatment: prevalence and risk factors for poor quality medicines in public facilities and private sector drug outlets in Enugu, Nigeria. PLOS ONE 2015;10: e0125577.

19. Adeloye D, Basquill C. Estimating the prevalence and awareness rates of hypertension in Africa: a systematic analysis. PLOS ONE 2014;9:e104300.

20. Kayima J, Wanyenze RK, Katamba A, et al. Hypertension awareness, treatment and control in Africa: a systematic review. BMC Cardiovasc Disord 2013;13:54.

21. Encyclopedia of the Nations. Nigeria. http://www.nationsencyclopedia. com/economies/Africa/Nigeria.html (accessed 2 Aug 2016).

22. World Health Organisation. Nigeria: WHO statistical profile, last updated 2015. http://www.who.int/gho/countries/nga.pdf?ua=1 (accessed 29 Jun 2016).

23. Sola AO, Chinyere OI, Stephen $\mathrm{AO}$, et al. Hypertension prevalence in an urban and rural area of Nigeria. J Med Sci 2013;4:149-54.

24. National Agency for Food and Drug, 2015. http://www.nafdac.gov.ng/ (accessed 26 Mar 2015).

25. United States Pharmacopeial reference standards for amlodipine besylate tablets. http://www.drugfuture.com/pharmacopoeia/usp35/ data/v35300/usp35nf30s0 m3575.html (accessed 2 Aug 2016)

26. United States Pharmacopeial reference standards for lisinopril tablets. http://www.pharmacopeia.cn/v29240/usp29nf24s0_m45568. html (accessed 2 Aug 2016). 